\title{
Formation of double-diffusive layers in the directional solidification of binary solution
}

\author{
Falin Chen \\ Institute of Applied Mechanics, National Taiwan University, Taipei 106, Taiwan ROC
}

Received 18 July 1996; accepted 2 December 1996

\begin{abstract}
The convective flows in the directional solidification of ammonium chloride solution cooling from below, categorized as the salt-finger convection, the plume convection and the bulk convection, have been found to be influential on the structure of resultant casting. Under some circumstances, as will be shown in the present study, the bulk convection will be replaced by the double diffusive convection in horizontally stratified layers; which will also cause a deleterious effect on the mechanical properties of the casting. The formation of the horizontal double diffusive layers (DDLs) turns out to be a result of the interaction between the vertical concentration gradient and the horizontal temperature gradient, both induced by the plume convection. The vertical concentration gradient is established due to the less concentrated fluid brought by the plumes to the top boundary and the horizontal temperature gradient is built-up due to the local cooling resulting from the plumes. Consequently, the DDL generates in the upper half of the bulk fluid region and then moves steadily downwards. As it reaches the melt/mush interface, the DDL structure breaks down, causing a slight remelting of the crystal of the mush. Results also show that the critical condition for the onset of DDL determined previously applies well for the present system. The DDL configuration can be closely related to the zonation in the magma chamber and the segregation of alloy casting.
\end{abstract}

\section{Introduction}

Since Copley et al. [1] conducted the experiment of directional solidification of aqueous ammonium chloride solution cooling from below, similar experiments have been carried out based on various points of view [2-10]. Theoretical analyses have also been performed to gain physical insights into the phenomena observed in the experiments [11-15]. In all these investigations, emphasis has been placed on the characteristics of the convective flows occurring during the solidification process, especially the salt-finger and the plume convection, the two major flows predominate the system. Under some circumstances, nevertheless, another major flow, the horizontal double-diffusive layers (DDLs) similar to those observed by, for example, Thorpe et al. [16] and Chen et al. [17], may also occur in the system and eventually predominate the convection of the system. Like the plume convection, the DDL has significant influence on the composition and structure of the resultant casting. 
Interestingly enough, the occurrence of DDL in the directional solidification of ammonium chloride solution has only been reported qualitatively by Magirl and Incropera [5], who conducted the experiments in a box of medium height. Otherwise, relevant report has been absent from most of the investigations. In the present study, we conducted a series of experiments to investigate the formation of DDL. Based on both qualitative observation and quantitative measurement, we are able to identify the physical mechanism driving the formation of DDL and to explain why the DDL was not observed by most of the previous experiments.

This paper is organized as follows. The experimental equipment and procedure is described in Section 2. A general observation of the convective flow concerning the formation of DDL is given in Section 3. A typical case study, which to some extent can be representative in terms of the characteristics of the formation of DDL for all the cases considered in the present study, is discussed in Section 4. The influences due to the bottom temperature and the height of the box on the formation of DDL are discussed in Sections 5 and 6, respectively. Finally, several concluding remarks are given in Section 7.

\section{Experimental equipment and procedure}

The experiments were performed in a test box similar to the one used in Chen et al. [8] except the revisions made as follows. The horizontal dimensions of the present box was $10.5 \times 10.5 \mathrm{~cm}^{2}$. The side walls were made of $2 \mathrm{~cm}$ thick plexiglass to enhance the insulation on the walls. Since during the experiment the side walls were also insulated with Styrofoam, the possible heat transfer from the side walls should accordingly be excluded. The top plate was made of brass and was provided with passages, through which the fluid from constanttemperature Lauda water-bath could circulate. The height of the box could be adjusted by changing the stand-set of the top plate. Each set of stand consists of four Plexiglass square columns of cross section area of $0.5 \times 0.5 \mathrm{~cm}^{2}$, which was placed inside the box (at corners) so that the top plate can be rested on the columns. Totally, seven sets of stand were made, ranging from 8 to $20 \mathrm{~cm}$ in height. A hole of $0.8 \mathrm{~cm}$ in diameter was drilled at the center of the top plate, through which the microscale conductivity-temperature (MSCT) probe [18] could move vertically.

The experiments started with a quiescent fluid of bulk temperature about $30^{\circ} \mathrm{C}$ and bulk concentration $28 \mathrm{wt} \%$. The temperature of the top boundary $T_{\mathrm{T}}$ was fixed at about $30^{\circ} \mathrm{C}$ for all the experiments and the temperature of the bottom boundary $T_{\mathrm{B}}$ could vary from about $-30-10^{\circ} \mathrm{C}$. During the experiment, the flow was visualized by either the shadowgraph or the schlieren technique. The time evolutions of the height of the mush, the height of the eutectic solid, the number of plumes, the height of the salt-fingers, and the position of each interface between DDLs were recorded. The $T_{\mathrm{T}}$ and $T_{\mathrm{B}}$ were monitored by the two thermocouples buried at the center of the boundaries. The MSCT probe was mounted on a three-axis stage controller to scan the vertical distributions of temperature and concentration at the horizontal center of the box. On a side wall, several thermocouples were inserted to monitor the vertical temperature distribution of the wall.

\section{General observation of formation of DDL}

Fig. 1 illustrates the flow activities of a $28 \mathrm{wt} \%$ $\mathrm{NH}_{4} \mathrm{Cl}$ solution cooling from below. The flow was visualized with a schlieren system. The $T_{\mathrm{B}}$ was $-18.7^{\circ} \mathrm{C}$, the $T_{\mathrm{T}}$ was $29.6^{\circ} \mathrm{C}$ and the height of the box $H$ was $10 \mathrm{~cm}$. Each picture is of $4 \mathrm{~cm}$ width, showing the central portion of the front view. The bottom of the picture is the bottom of the box.

As the experiment began, crystallization started to form at the bottom of the box, accompanied by the occurrence of the salt-finger convection. Fig. 1a shows the salt-finger convection and the mushy zone (dark region at bottom) when the experiment had been running for about five minutes ( $t=5 \mathrm{~min})$ and the thickness of the mush was about $0.4 \mathrm{~cm}$. At $t=37 \mathrm{~min}$ (Fig. 1b) the salt-finger convection became more vigorous and the plume convection also prevailed, the thickness of mush had increased to about $2 \mathrm{~cm}$. At $t=88 \mathrm{~min}$ (Fig. 1c) the thickness of mush was about $3.1 \mathrm{~cm}$, the salt-finger convection 

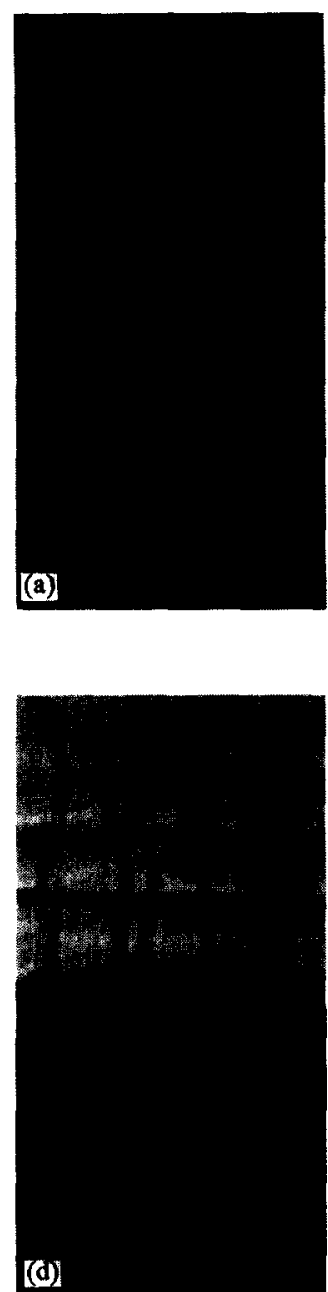
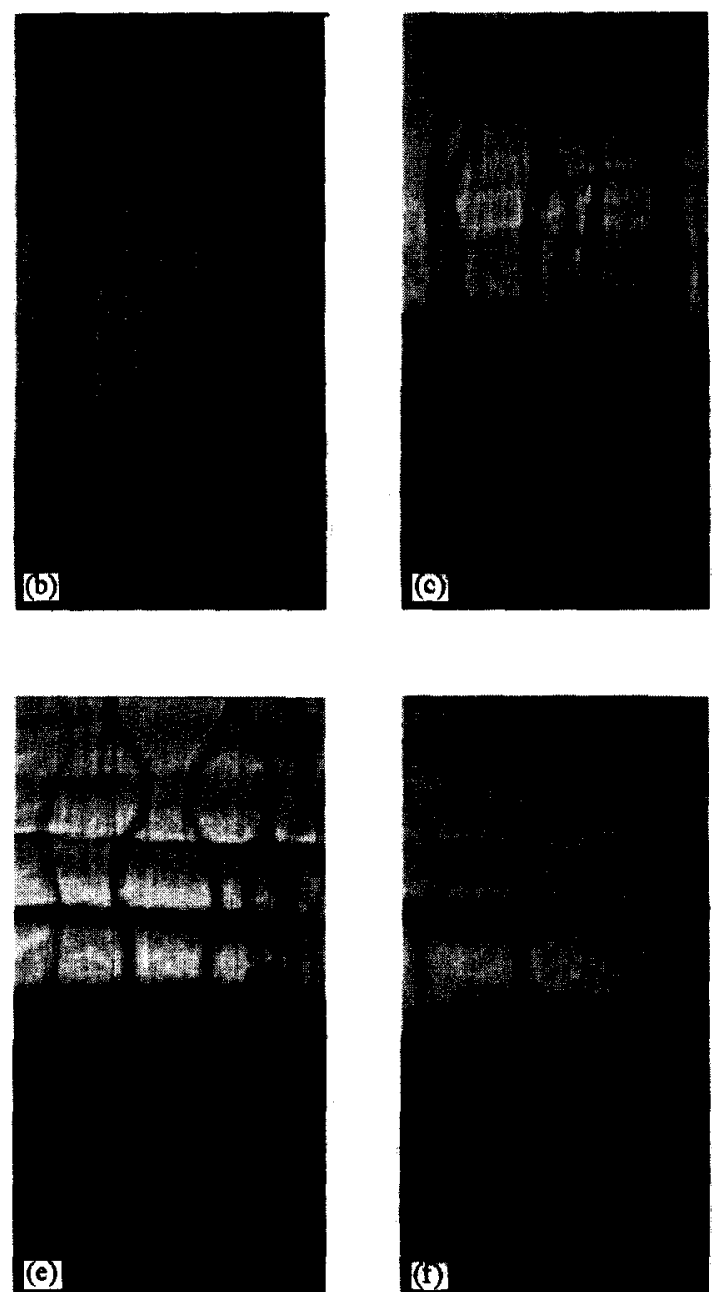

Fig. 1. Shadows of the convective flows visualized with the schlieren technique. The experimental conditions are $T_{\mathrm{T}}=29.6^{\circ} \mathrm{C}$, $T_{\mathrm{B}}=-18.7^{\circ} \mathrm{C}$ and $H=10 \mathrm{~cm}$. Each picture shows the $4 \mathrm{~cm}$ wide central portion of the test section from the front view and the bottom of the picture is exactly the bottom of the box: (a) $t=5 \mathrm{~min}$; (b) $t=37 \mathrm{~min}$; (c) $t=88 \mathrm{~min}$; (d) $t=110 \mathrm{~min}$; (e) $t=121 \mathrm{~min}$; (f) $t=159 \mathrm{~min}$.

disappeared, the prevailing of the plume convection remained, and several horizontal DDLs existed simultaneously. At $t=110 \mathrm{~min}$ (Fig. 1d) the number of plumes obviously decreased whereas the DDL structure became more organized. At $t=121 \mathrm{~min}$ (Fig. 1e) the DDL still prevailed and the number of plumes decreased further. At $t=159$ min (Fig. If) some of the DDLs disappeared and the plume convection decayed continuously.

From observations of the above case and other cases considered in the present study, several fea- tures concerning the DDL formation can be delineated in the following. In general, the first layer interface between DDLs was usually observed in the upper half of the bulk fluid. Then the layer interface moved downwards continuously and new DDL generated from above. The DDL broke down as it reached the melt/mush interface. The convection within the DDL was generally three-dimensional, the fluid circulated like a helix from below. The flow pattern of the plume was not interfered with the presence of the DDL as it penetrated 
through the layer-interface. The configuration of DDL, similarly, was not influenced by the penetration of the plume either. The DDL had never formed in the salt-finger region.

\section{Case study: $T_{\mathrm{B}}=-9.8^{\circ} \mathrm{C}, T_{\mathrm{T}}=29.6^{\circ} \mathrm{C}$, $H=10 \mathrm{~cm}$}

The flow characteristics concerned with the formation of $\mathrm{DDL}$ of this case is to some extent representative for all the cases considered in the present study. We give in this section the details of the experimental results of the present case, with which a thorough understanding about the formation of DDL in connection with the solidification can be obtained.

\subsection{Flow characteristics}

Fig. 2 gives a complete picture of the time evolutions of the position of the melt/mush interface

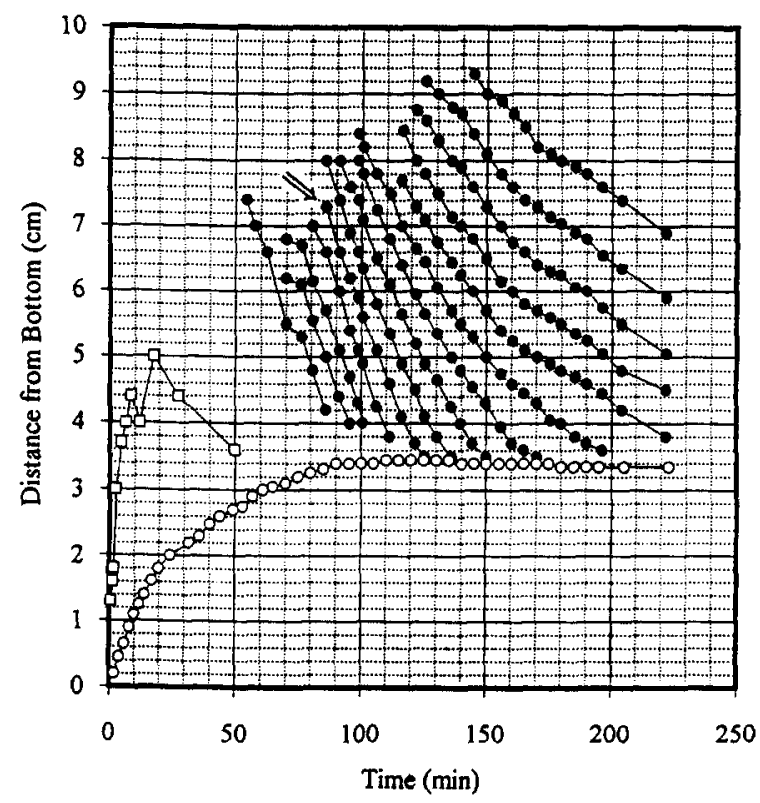

Fig. 2. Time evolutions of the positions of the melt/mush interface (blank circles), the top of the salt-finger (squares) and the layer interface (solid circles) for the case of Section 4. The arrow points out the position of the fifth interface, which is employed as an example for the explanation of the remelting of the mush in Section 4.2 (blank circles), the top of the salt-finger (squares), and the positions of the layer interfaces (solid circles). At the beginning of the experiment the mush grew continuously, the salt-finger increased its vertical extent, reached the maximum at $t \approx 19 \mathrm{~min}$ and then decreased. At $t \approx 50 \mathrm{~min}$, the salt finger virtually vanished and, a couple of minutes later, the first layer interface was observed at $h \approx 7.4 \mathrm{~cm}$. Afterwards, the first layer interface moved downwards continuously, and two new layer interfaces appeared at $h \approx 6.8$ and $6.2 \mathrm{~cm}$ when $t \approx 70 \mathrm{~min}$. At the same time the first layer interface reached at $h \approx 5.5 \mathrm{~cm}$. New layer interfaces generated continuously from the top and the existing layer interfaces moved downwards to the melt $/$ mush interface. At $t=100 \mathrm{~min}$, for example, there were seven layer interfaces in the system and the layer thickness was about $0.7 \mathrm{~cm}$ in average. At $t=150 \mathrm{~min}$, for another example, there were eight layer interfaces in the system and the averaged layer thickness was about $0.8 \mathrm{~cm}$.

It is noteworthy that at $t \approx 125 \mathrm{~min}$ the lowest layer interface coalesced with the melt/mush interface and then disappeared. About 15 min later another layer interface reached and disappeared. The collapse of DDL led to the remelting of the mush; the reason causing the remelting will be discussed in Section 4.2. The propagating speed of the layer interface in different time domains can also be estimated from Fig. 2. By and large, the averaged propagating speed was about $0.075 \mathrm{~cm} / \mathrm{min}$ in $50 \mathrm{~min}<t<100 \mathrm{~min}$, about $0.064 \mathrm{~cm} / \mathrm{min}$ in $100 \mathrm{~min}<t<150 \mathrm{~min}$ and about $0.043 \mathrm{~cm} / \mathrm{min}$ in $150 \mathrm{~min}<t<200 \mathrm{~min}$. Qualitatively, the layer interface moved steadily downwards at the beginning of its formation. As time went on, the propagating speed decreased slightly until it disappeared. On the other hand, the layer thickness (or the distance between two layer interfaces) did not change significantly with time although a small temporal increment was still visible.

It is also found as a general rule that the onset of DDL occurs approximately when the number of plumes reaches the maximum. This can be seen from Fig. 3 that the number of plumes reached the maximum at $t \approx 55 \mathrm{~min}$, about the same time with the onset of DDL (Fig. 2). This general rule implies that the vigor of plume convection has a close 


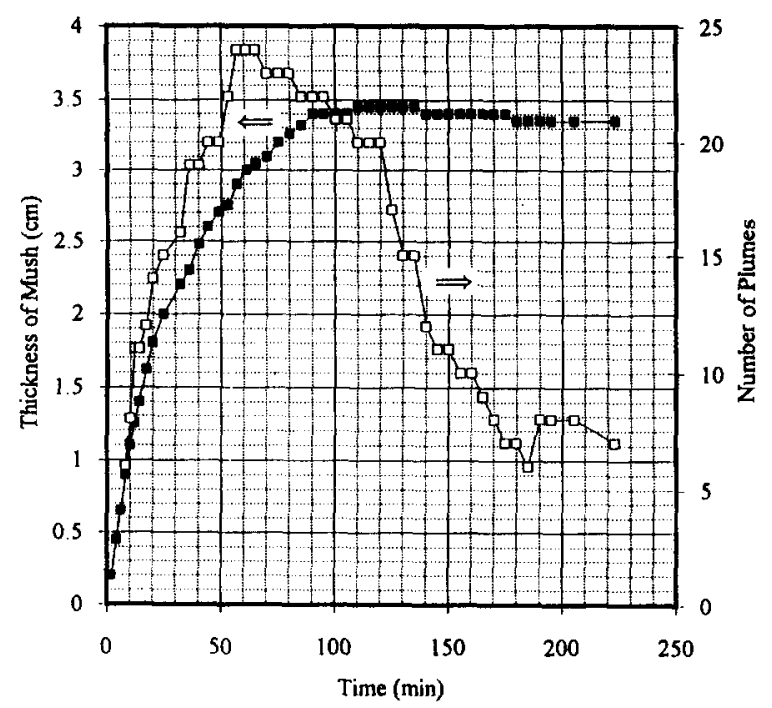

Fig. 3. Time evolutions of the thickness of mush (black squares) and the number of plume (blank squares) for the case of Section 4.

relation with the onset of DDL, which will be discussed in Section 4.3. The growth and the remelting of the mush can be more clearly seen from Fig. 3. In about $100 \mathrm{~min}$ after the first layer interface coalesced with the melt/mush interface, the mush remelted about $0.15 \mathrm{~cm}$ in thickness.

\subsection{Vertical distributions of temperature and concentration}

Fig. 4a and Fig. 4b illustrate the vertical distributions of temperature and concentration, respec-

Fig. 4. The vertical temperature distribution (a) and the vertical concentration distribution (b) at different times for the case of Section 4 . The curves are divided into the bulk fluid region and the DDL region by a coarse curve, which is determined by observing the corresponding flow pictures taken during the experiment. The salt-finger region appears only as a small region sitting below the solid circle of curve No. 1. The time for each curve is shown in the following: curve Nos. (1) $t \approx 40 \mathrm{~min}$; (2) $t \approx 51 \mathrm{~min}$; (3) $t \approx 62 \mathrm{~min}$; (4) $t \approx 73 \mathrm{~min} ;$ (5) $t \approx 83 \mathrm{~min}$; (6) $t \approx 93 \mathrm{~min} ;(7) t \approx 104 \mathrm{~min} ;(8) t \approx 111 \mathrm{~min} ;(9) t \approx 117 \mathrm{~min} ;(10)$ $t \approx 128 \mathrm{~min} ;(11) t \approx 140 \mathrm{~min} ;(12) t \approx 151 \mathrm{~min} ;(13) t \approx 168 \mathrm{~min} ;$ (14) $t \approx 183 \mathrm{~min}$; (15) $t \approx 226 \mathrm{~min}$. Note that in (b), the blank circles of curves Nos. 6 and 10 correspond with the generation and the break down of the fifth layer-interface shown in Fig. 2. tively, at different times. These measurements were made with the MSCT probe fixed on a computercontrolled XYZ-positioner. The probe was programmed to move along the vertical axis at the horizontal center of the box. The moving speed of the probe was about $0.05 \mathrm{~cm} / \mathrm{s}$, so that it took about $3 \mathrm{~min}$ or less to finish a scan. The scan was
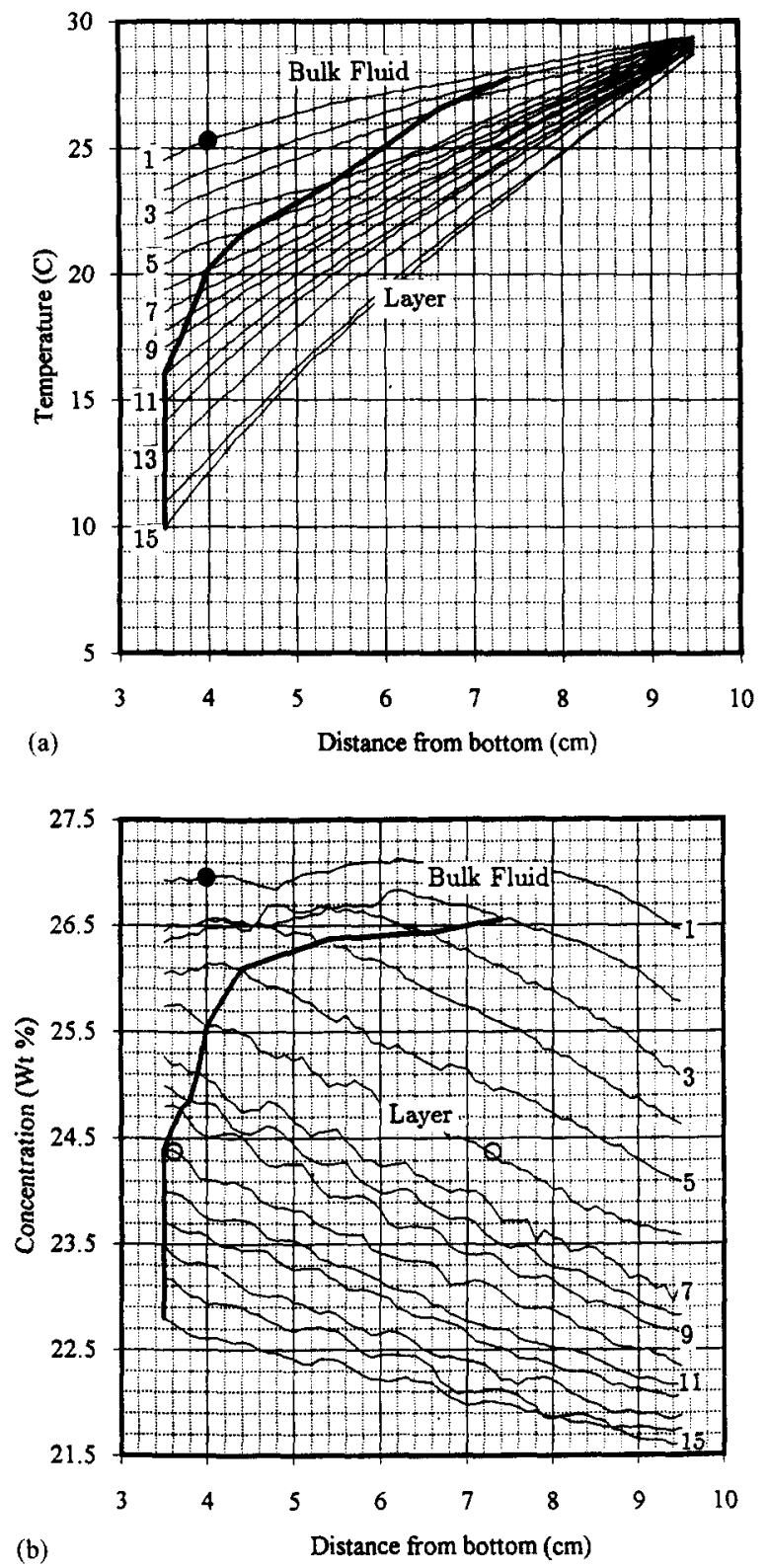
carried out approximately every ten minutes, starting from the top of the box and ending at the vicinity of the melt/mush interface. There are 15 curves shown in each figure and the scan started from $t \approx 40 \mathrm{~min}$ (curve No. 1) when the onset of DDL was about to occur. With the aids from the observation of the flow evolution, we were able to divide these curves into the bulk fluid region and the layer region. Note that the salt-finger region in the figure only appeared as a small region sitting below the solid circle of the curve No. 1 .

It is seen from Fig. 4a that the temperature decreased vertically from $T_{\mathrm{T}} \approx 29^{\circ} \mathrm{C}$ to a value decreasing with time. The vertical temperature gradient $\nabla_{\mathrm{v}} T$ was invariably positive in both regions and increased with time monotonically. Accordingly, the $\nabla_{\mathrm{v}} T$ stabilized the flow in both regions. For the vertical concentration distribution (Fig. 4b), curve No. 1 showed that $\nabla_{\mathrm{v}} C$ near the top was negative while the concentration in the bulk fluid region was relatively more uniform. The negative $\nabla_{\mathrm{v}} C$ was a result of the less-concentrated fluid brought by the plumes into the upper region, leading to the decrease of concentration from the top. The value of $\nabla_{\mathrm{v}} C$ became more negative with time since the number of plumes increased with time. At $t \approx 51 \mathrm{~min}$ (curve No. 2) the first layer interface appeared in the upper part of the bulk fluid. The $\nabla_{\mathrm{v}} C$ became more negative with further time; until at $t \geqslant 83 \mathrm{~min}$ (curves No. 5 and others) it turned into less negative. Note that the MSCT measurements of both temperature and concentration showed some small fluctuations, which might be due to the disturbance caused by the convective flow on the probe. However, the concentration stratification of the DDL was clearly reflected by the measurements shown in curves No. 7-15.

Regarding the remelting of the mush as the DDL breaks down at the melt/mush interface, the physical explanation is provided in the following. As the DDL formed in the upper part of the bulk fluid, the concentration of the fluid within the layer was low and remained vitually unchanged during its propagating downwards because of the small concentration diffusivity across the layer interface. As the lowest layer interface broke down, the less-concentrated fluid of the DDL interacted with the mushy zone, resulting in the remelting of the crystal of the mush and thus the decrease of the thickness of mush. To explain this, we focus on the fifth layer interface of Fig. 2 (pointed out with an arrow), which showed that the layer interface generated at $t \approx 85 \mathrm{~min}$ and $h \approx 7.3 \mathrm{~cm}$ and reached the melt $/$ mush interface at $t \approx 125 \mathrm{~min}$ and $h \approx 3.6 \mathrm{~cm}$, corresponding with the blank circles of curve No. 6 at $h \approx 7.3 \mathrm{~cm}$ and of curve No. 10 at $h \approx 3.6 \mathrm{~cm}$ of Fig. $4 \mathrm{~b}$. At the blank circle of curve No. 6, the layer interface generated; at the blank circle of curve No. 10 , the layer interface broke down. On both blank circles, the concentration remained virtually the same, approximately $24.3 \mathrm{wt} \%$, supporting well the above scenario.

\subsection{Onset of double-diffusive layer}

After the plumes emerged from the interior of the mush, the vertical concentration gradient gradually became more negative due to the less concentrated fluid brought by the plumes into the top of the bulk fluid region. The vertical temperature gradient, instead of being influenced by the cold plumes, became more positive due to the enforced high temperature at the top boundary $\left(T_{\mathrm{T}} \approx 30^{\circ} \mathrm{C}\right)$ and the upward moving melt/mush interface on which supercooling occurred. Consequently, both the positive temperature and negative concentration vertical gradients intended to stabilize the flow in the bulk fluid, which was randomly driven by the disturbance of the entraining plumes. Under such a well-stabilized environment, the onset of the DDL was made possible by the horizontal temperature gradient $\nabla_{\mathrm{h}} T$ due to cold plumes. The following quantitative evidences support this scenario.

Fig. 5 illustrates the time evolutions of the wall temperature (solid curve) and the solution temperature at the horizontal center of the box (curve with markers) at three positions: $h=6.5,5.5$ and $4.5 \mathrm{~cm}$. Note that the horizontal distribution of the plumes usually is uniform and the thickness of layer is also uniform in the horizontal sense, the $\nabla_{\mathrm{h}} T$ of the system can be accounted for by the temperature difference between the wall and the horizontal center of the box $\left(\Delta T_{\mathrm{h}}\right)$. The onset of layer occurred at $t \approx 50 \mathrm{~min}$ and $h \approx 7 \mathrm{~cm}$. At $h=6.5 \mathrm{~cm}$ (the first couple of curves from the top), the temperatures at the wall and the center were virtually the same at 


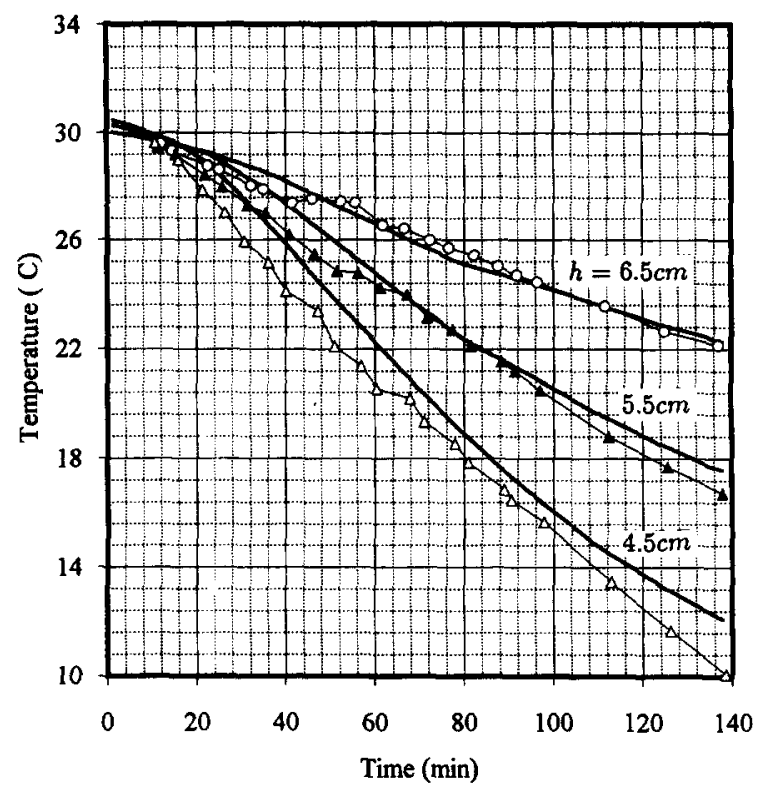

Fig. 5. Time evolutions of the temperature at the side wall (solid curve) and at the horizontal center (curve with markers) at different vertical positions as shown.

the beginning of the experiment and both decreased with time. Their difference, however, became larger as time went on, lying within $1{ }^{\circ} \mathrm{C}<\Delta T_{\mathrm{h}}<2^{\circ} \mathrm{C}$ before the onset, and virtually vanished after the onset because the convection in the DDL homogenized the temperature horizontally. A similar case applies for the temperatures at $h=5.5 \mathrm{~cm}$ where the onset of DDL occurred at $t \approx 60 \mathrm{~min}$ and at $h=4.5 \mathrm{~cm}$ the onset occurred at $t \approx 70 \mathrm{~min}$.

Thorpe et al. [16] examined experimentally the onset of DDL in a narrow tank and concluded that the critical condition for the onset of DDL in terms of thermal and solutal Rayleigh numbers, $R_{\mathrm{t}}$ and $R_{\mathrm{s}}$, are of the following relation

$R_{\mathrm{t}}=0.06\left(R_{\mathrm{s}}\right)^{5 / 6}$,

where

$R_{\mathrm{s}}=\frac{g \beta\left(\Delta C_{\mathrm{v}} / \Delta z\right) D^{4}}{v \kappa_{\mathrm{s}}}, \quad R_{\mathrm{t}}=\frac{g_{x} \Delta T_{\mathrm{h}} D^{3}}{v \kappa_{\mathrm{t}}}$

in which $g$ is the gravitational acceleration, $\alpha$ and $\beta$ the expansion coefficients due to temperature and concentration, respectively, $v$ the kinematic viscosity, $D$ the characteristic horizontal length, and $\kappa_{\mathrm{t}}$ and $\kappa_{\mathrm{s}}$ the thermal and solutal diffusivities, respectively. The $\Delta C_{\mathrm{v}}$ was measured across the region $6 \mathrm{~cm} \leqslant h \leqslant 8 \mathrm{~cm}$ for the layer region in which the onset of layer usually occurred and the $\Delta T_{\mathrm{h}}$ was the temperature difference in the horizontal direction between the horizontal center of the box and the wall. In the present case, the $R_{\mathrm{s}}$ is approximately $4 \times 10^{9}$ when $D=5 \mathrm{~cm}$ (the distance between the wall and the horizontal center) were considered. As a result, the critical value of $R_{\mathrm{t}}$ was about $5.9 \times 10^{6}$; or equivalently the critical $\Delta T_{\mathrm{h}}$ was $2.02^{\circ} \mathrm{C}$, which was in very good agreement with the present result. A similar conclusion can also be reached when the critical condition for a wide tank [17] in terms of the critical thermal Rayleigh number

$R_{\mathrm{tw}}=\frac{g \alpha \Delta T_{\mathrm{h}} d^{3}}{v \kappa_{\mathrm{t}}}=420 L^{2}$

is considered, where $L$ is the width of the tank (here is $5 \mathrm{~cm}$, center to wall) normalized with respect to the layer thickness $d$ (here is about $0.7 \mathrm{~cm}$ ).

Another comparison can be made from the data shown in Fig. 4b. It has been shown that at the onset of DDL we have $R_{\mathrm{s}} \approx 4 \times 10^{9}$, giving $\Delta C_{\mathrm{v}} /$ $\Delta \mathrm{z} \approx 0.48 \mathrm{wt} \% \mathrm{~cm}^{-1}$. This value agrees very well with the measurement shown in Fig. $4 \mathrm{~b}$ that, before the onset of DDL, curve No. 2 gives $\Delta C_{\mathrm{v}}$ / $\Delta \mathrm{z} \approx 0.41 \mathrm{wt} \% \mathrm{~cm}^{-1}$, and after the onset, curve No. 3 gives $\Delta C_{\mathrm{v}} / \Delta \mathrm{z} \approx 0.5 \mathrm{wt} \% \mathrm{~cm}^{-1}$. The physical properties of the solution can be obtained from, for example, Chen et al. [19]. We noted that in Fig. 4a and Fig. $4 \mathrm{~b}$ the scan of each curve took roughly 3 min, while in the mean time the overall values of both $\Delta T_{\mathrm{v}}$ and $\Delta C_{\mathrm{v}}$ are changing. The above values of $\Delta C_{\mathrm{v}}$ accordingly can be underestimated. We therefore examined the curves No. 5-7, on which the changes of $\Delta T_{\mathrm{v}}$ and $\Delta C_{\mathrm{v}}$ are the largest, and found that the time-lag due to scanning leads to approximately a $2 \%$ error in $\Delta T_{\mathrm{v}}$ and a $7 \%$ error in $\Delta C_{v}$, which indeed do not influence the present conclusion.

\section{Influence of $T_{\mathrm{B}}$}

Experiments with various $T_{\mathrm{B}}$, ranging from approximately -30 to $9.5^{\circ} \mathrm{C}$, have been carried out when $T_{\mathrm{T}} \approx 30^{\circ} \mathrm{C}$ and $H=10 \mathrm{~cm}$. Although the top 
boundary and $H$ are different from the experimental setup of Chen et al. [19], the time evolutions of both the growth of mush and the number of plumes in both studies are quite similar. Generally, the growth of mush (see Fig. 6 of Chen et al. [19]) depends heavily upon the value of $T_{B}$ and the largest growth rate occurs when $T_{\mathrm{B}}$ close to the temperature of the eutectic solid $T_{\mathrm{S}} \approx-16^{\circ} \mathrm{C}$, and is larger for $T_{\mathrm{B}}<T_{\mathrm{S}}$ than for $T_{\mathrm{B}}>T_{\mathrm{S}}$. The time evolution of plume number (see Fig. 7 of Chen et al. [19]) is also influenced significantly by $T_{\mathrm{B}}$. The time for the number of plumes reaching the maximum is shorter for lower $T_{B}$. Since the onset of DDL occurs when the number of plume reaches the maximum, the time for the onset of DDL is accordingly shorter for lower $T_{\mathrm{B}}$. The layer thickness (Fig. 6), however, is relatively insensitive to the variation of $T_{\mathbf{B}}$; which generally fluctuates between 0.6 and $1 \mathrm{~cm}$ all the time. According to Chen et al. [17], for a $\Delta T_{\mathrm{h}}=2^{\circ} \mathrm{C}$ and $\nabla_{\mathrm{v}} \mathrm{C}=$ $0.43 \mathrm{wt} \% \mathrm{~cm}^{-1}$, the larger thickness should be of the order of $0.62 \mathrm{~cm}$, which agrees with the present result ricely.

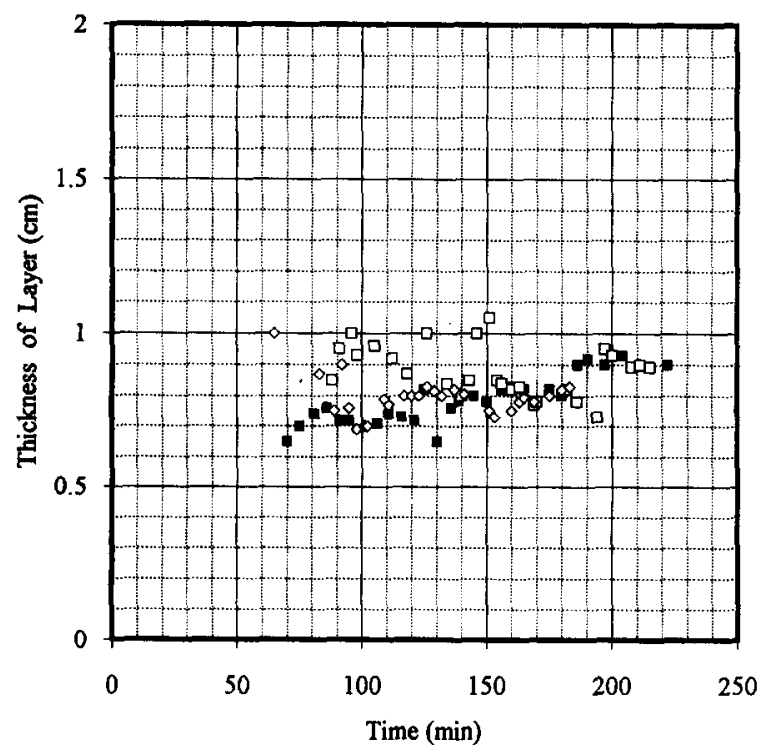

Fig. 6. Time evolutions of the averaged thickness of layer for $T_{\mathrm{B}}=0.5^{\circ} \mathrm{C}$ (blank squares), $T_{\mathrm{B}}=-9.8^{\circ} \mathrm{C}$ (solid squares), and $T_{\mathrm{B}}=-19.1^{\circ} \mathrm{C}$ (blank diamonds) when $T_{\mathrm{T}} \approx 29.5^{\circ} \mathrm{C}$ and $H=10 \mathrm{~cm}$.

\section{Influence of the height of the box $H$}

In all the previous experiments, only Magirl and Incropera [5] had observed the formation of DDL, who conducted the experiments in a finite box of $H \approx 10 \mathrm{~cm}$, being lower than the test boxes used in other studies. We therefore suspect that the formation of DDL can be influenced by the height of the box. To examine this, a series of experiments were conducted for $T_{\mathrm{B}} \approx-30^{\circ} \mathrm{C}, T_{\mathrm{T}} \approx-30^{\circ} \mathrm{C}$ and $8 \mathrm{~cm} \leqslant H \leqslant 20 \mathrm{~cm}$. We present in Fig. 7 the time evolution of the number of plumes for the four cases $H=8,10,12$ and $14 \mathrm{~cm}$, showing that the time evolution of the plume number is essentially insignificantly influenced by $H$.

Nevertheless, we found that the influence of $H$ on the DDL formation becomes effective by way of the stability of the plume. Since the cold and less concentrated plume rose through a bulk fluid of positive $\nabla_{\mathrm{v}} T$ and negative $\nabla_{\mathrm{v}} C$, the plume lost the stability and eventually broke down in the bulk fluid [20]. As we observed in all the experiments considered, the plumes reached the top boundary

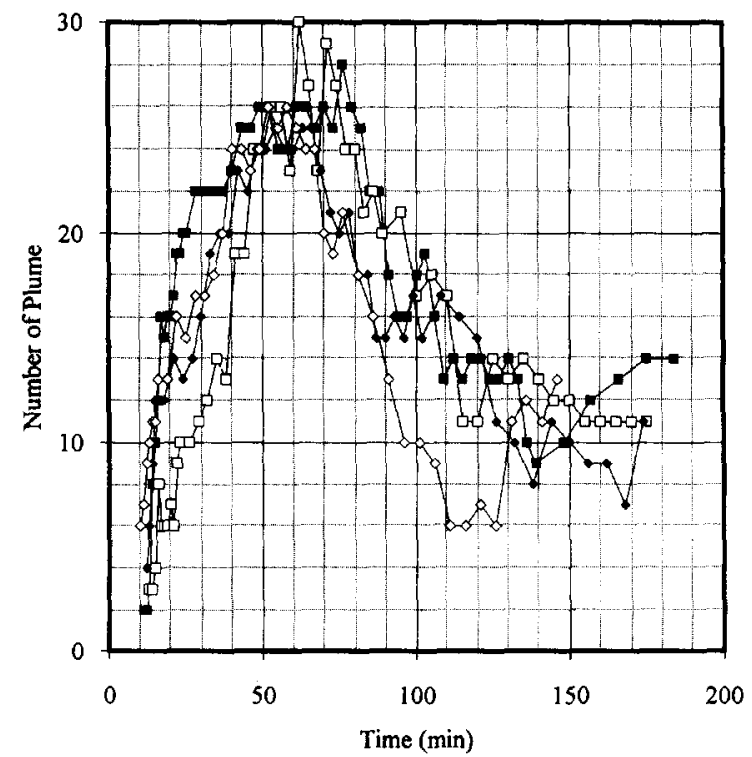

Fig. 7. Time evolutions of the number of plume for $T_{\mathrm{B}} \approx-29^{\circ} \mathrm{C}, T_{\mathrm{T}} \approx 30^{\circ} \mathrm{C}$ and $H=14 \mathrm{~cm}$ (solid squares), $12 \mathrm{~cm}$ (blank squares), $10 \mathrm{~cm}$ (solid diamonds) and $8 \mathrm{~cm}$ (blank diamonds). 
without significantly mixing with the ambient fluid when $H \leqslant 18 \mathrm{~cm}$. If the plume rached the top before it collapsed, the negative $\nabla_{\mathrm{v}} C$ was built up, as shown in curve No. 2 of Fig. 4b; which turned out to be a necessary condition for the formation of DDL. Nevertheless, if the plume collapsed before it reached the top, the less concentrated fluid of the plume mixed up with the solute-rich bulk fluid and the negative $\nabla_{\mathrm{y}} C$ was never been established. This explains why the DDL has never been observed in the present study when $H=20 \mathrm{~cm}$, and in other experiments with even larger $H$.

\section{Concluding remarks}

A systematic investigation has been implemented for studying the formation of double diffusive layers during the directional solidification of aqueous ammonium chloride solution cooling from below. From the above results and discussion, several conclusive remarks can be made.

1. The formation of DDL is a result of the interaction between the negative vertical concentration gradient and the horizontal temperature gradient, both resulting from the plume convection.

2. A new DDL generates in the upper part of the bulk fluid. After its formation, it moves steadily downwards to the melt/mush interface; on which, it breaks down and the less-concentrated fluid within it induces the remelting of the crystal of the mush.

3. The formation of DDL occurs when the number of plumes reaches the maximum. Both the critical conditions of the onset of DDL developed for a narrow tank and for a wide tank apply well for the present system.

4. The value of $T_{B}$ is influential on the evolution of number of plumes and thus on the onset-time of DDL, but is insignificant to the thickness of DDL.

5. The condition $H<20 \mathrm{~cm}$ is necessary for the formation of DDL. Because under this condition the plume reaches the top before it collapses and a negative vertical concentration gradient can be established.

6. The DDL is never formed in the salt-finger region because the two gradients mentioned in remark No. 1 do not exist.
7. The value of $H$ does not seem to affect the evolution of the number of plumes.

Finally, it is noted that the formation of DDL will cause deleterious effects on the mechanical strength of the resultant casting because the interface between the density-stratified zones (or the DDLs) is perpendicular to the grain boundaries of the casting, which are in the same direction of solidification as well as the applied centrifugal force on the casting (such as a turbine blade). As a result, like the occurrence of plumes which corresponds with the formation of freckles, the occurrence of DDL will result in the horizontal zonations of the casting and in turn greatly diminishes the superiority in terms of the increased creep rupture strain and the improved thermal fatigue behavior of the casting resulting from the directional solidification process. Fotunately, since the formation of DDL has been proved to be merely a consequence of the plume convection, one needs only to prohibit the occurrence of plume then the formation of DDL can be also prevented. The critical condition for the onset of plume convection has been determined by Chen et al. [8].

\section{Acknowledgements}

The phenomenon of the formation of DDL was first observed by the author when he, as a $\mathrm{PhD}$ student in University of Arizona, performed a testrun in the box of $H>30 \mathrm{~cm}$ for the experimental work of Chen and Chen [2]. The reason for the formation of DDL, as pointed out by Professor C.F. Chen, the thesis adviser of the author, was the strong convective cooling on the side walls due to the ventilation of the laboratory. Later, during the experimental work of Chen, Lu and Yang [8], the author found that the DDL will also occur in a box of $H<10 \mathrm{~cm}$ although the side walls were well insulated. This experiment was also conducted in DAMTP of University of Cambridge when the author was on a sabbatical leave. During the visit the stimulus discussion with Professor H.E. Huppert turned out to be helpful for the final work done in the IAM of National Taiwan University. The help from Messrs. T.L. Yang, T.I. Liao and J.R. Chen for preparing the experiments and/or 
collecting the data is appreciated. The financial support for the present work through grant NSC84-2212-E-002-055 is gratefully acknowledged.

\section{References}

[1] S.M. Copley, A.F. Giamei, S.M. Johnson and M.F. Hornbecker, Metall. Trans. 1 (1970) 2193.

[2] C.F. Chen and F. Chen, J. Fluid Mech. 227 (1991) 567.

[3] S. Tait, K. Jahrling and C. Jaupart, Nature 359 (1992) 406.

[4] S. Tait and C. Jaupart, J. Geophys. Res. 97 (1992) 6735.

[5] C.S. Magirl and F.P. Incropera, J. Heat Transfer ASME Trans. C 115 (1993) 1036.

[6] H.E. Huppert and M.A. Hallworth, J. Crystal Growth 130 (1993) 495.

[7] A. Hellawell, J.R. Sarazin and R.S. Steube, Phil. Trans. Roy. Soc. London A 345 (1993) 507.

[8] F. Chen, J.W. Lu and T.L. Yang, J. Fluid Mech. 276 (1994) 163.
[9] M.G. Worster and R.C. Kerr, J. Fluid Mech. 269 (1994) 22.

[10] C.F. Chen, J. Fluid Mech. 293 (1995) 81.

[11] A.C. Fowler, IMA J. Appl. Math. 35 (1985) 159.

[12] W.D. Bennon and F.P. Incropera, Int. J. Heat Mass Transfer 30 (1987) 2161.

[13] M.G. Worster, J. Fluid Mech. 237 (1992) 649.

[14] G. Amberg and G.M. Homsy, J. Fluid Mech. 252 (1993) 79.

[15] P.W. Emms and A.C. Fowler, J. Fluid Mech. 262 (1994) 111.

[16] S.A. Thorpe, P.K. Hutt and R. Soulsby, J. Fluid Mech. 38 (1969) 375.

[17] C.F. Chen, D.G. Briggs and R.A. Wirtz, Int. J. Heat Mass Transfer 14 (1971) 57.

[18] M.J. Head, The use of miniature four-electrode conductivity probes for high resolution measurement of turbulent density or temperature variations in salt-stratified water flows, PhD Dissertation, University of California, San Diego (1983).

[19] F. Chen, T.L. Yang and J.W. Lu, J. Appl. Phys. 74 (12) (1993) 7531.

[20] I.A. Eltayeb and D.E. Loper, J. Fluid Mech. 267 (1994) 251. 